\title{
Unterstützte Kommunikation (UK) mit der iPad-App MetaTalkDE
}

\author{
Augmentative and Alternative Communication (AAC) with the iPad-App MetaTalkDE
}

\author{
Im Rahmen einer deutschlandweiten Onlinebefragung wurde \\ die Nutzung der UK-App MetaTalkDE erfasst. Die 115 Teilneh- \\ mer gaben an, die App auf vielfältige Weise als Kommunika- \\ tionshilfe zu nutzen, insbesondere das iPad erfreute sich auf- \\ grund seiner Multifunktionalität großer Beliebtheit.
}

\section{Hintergrund \\ $\nabla$}

Manche Menschen können sich aufgrund von angeborenen Schädigungen, wie z.B. geistiger Behinderung oder erworbenen Schädigungen, nicht erfolgreich lautsprachlich äußern. Beispielsweise ist bei Schlaganfallpatienten häufig das Sprachzentrum vorübergehend oder langfristig beeinträchtigt. Ziel des Fachgebiets der Unterstützten Kommunikation (UK) ist es, die Kommunikationsmöglichkeiten dieser Menschen zu verbessern [1]

Vor ca. 25 Jahren bestand für diese Personengruppe in erster Linie die Möglichkeit, sich über körpereigene Kommunikationsformen, wie beispielsweise Handzeichen, mitzuteilen. Heute gibt es viele verschiedene Wege, ohne Lautsprache zu kommunizieren. Besonders externe Kommunikationsformen, wie elektronische Hilfsmittel, haben im letzten Vierteljahrhundert aufgrund des technischen Fortschritts deutlich zugenommen.

Seit Anfang 2010 gibt es das iPad, das sich von spezifischen Kommunikationshilfen durch vielfältige Anwendungsmöglichkeiten und Funktionen abhebt. Obwohl es ursprünglich nicht als Kommunikationshilfe gedacht war, kamen bereits im Herbst 2010 erste UK-Anwendungen (Apps) für das iPad auf den Markt. Eine dieser Apps ist die iPad-App MetaTalkDE (Kitzinger, 2010). Zusammen mit dem iPad dient sie mittlerweile vielen nicht oder kaum sprechenden Menschen in Deutschland als Kommunikationshilfe [2]. Bisher ist aber nur wenig über die Nutzergruppe und deren Nutzungsverhalten bekannt. Im Rahmen der Staatsexamensarbeit wurde daher eine deutschlandweite Onlinebefragung mit dem Ziel durchgeführt, diese Forschungslücke zu füllen und Möglichkeiten für weitere Verbesserungen und Anpassungen der App zu finden.

\section{MetaTalkDE}

MetaTalkDE ist eine symbolbasierte App mit Schriftoption, die aktuell 180 Euro kostet. Es gibt sie in unterschiedlichen Varianten. In Abhängigkeit von den Rastergrößen $5 \times 9,4 \times 7$ und $3 \times 5$ und der Wortschatzgröße von 2500 , 1500 und 1000 Wörtern, besteht die Bildschirmoberfläche aus 45, 28 oder 15 Tasten. Bei den Varianten mit den Rastergrößen $5 \times 9$ und $4 \times 7$ gibt es zusätzliche Funktionen, wie zum Beispiel Grammatik-Popups [3].

\section{Zielsetzung \\ $\nabla$}

Ziel der deutschlandweiten Nutzerbefragung war eine „Positionsbestimmung“ der App und damit auch des iPads als Kommunikationshilfe. Dazu wurden soziodemografische Angaben der Nutzer der App bzw. des iPads erfragt, um herauszufinden, wie sich die Nutzergruppe zum Beispiel bzgl. des Alters zusammensetzt. Des Weiteren sollte mithilfe von Informationen zur Nutzung der App in Erfahrung gebracht werden, welche Funktionen der App wie häufig genutzt werden. Bereits bekannt war, dass das iPad als Kommunikationshilfe u.a. aufgrund seiner vielfältigen Funktionen und Möglichkeiten sehr beliebt ist. Gründe, warum es außerdem als solche verwendet wird, sollten mithilfe der Befragung eruiert werden.

\section{Methodik \\ $\nabla$}

Es wurde eine Nutzerbefragung in Form einer deutschlandweiten Onlineumfrage im Zeitraum von knapp 3 Monaten durchgeführt. Die Befragung richtete sich je-

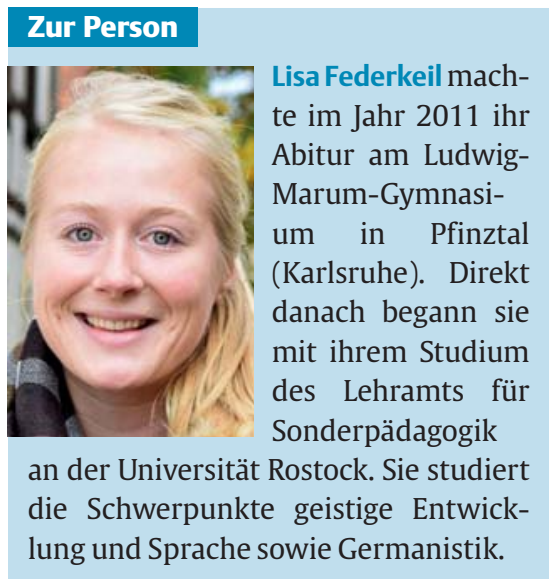

\section{Zum Studiengang}

Der Studiengang Lehramt für Sonderpädagogik wird am Institut für Sonderpädagogische Entwicklungsförderung (ISER) an der Universität Rostock mit den 4 Förderschwerpunkten Lernen, emotionale und soziale Entwicklung, Sprache sowie geistige Entwicklung angeboten. Von diesen müssen 2 Schwerpunkte sowie schwerpunktübergreifende Aspekte studiert werden. Außerdem umfasst der Studiengang die Bereiche Erziehungswissenschaften, ein allgemeinbildendes Fach oder Grundschulpädagogik sowie mehrere Praktika. Zentrale Kompetenzbereiche, die die Studierenden erwerben, sind Unterrichten, Erziehen, Diagnostizieren und Fördern, Innovieren und die Gestaltung einer inklusiven Schule.

doch nicht nur an die eigentlichen MetaTalkDE-Nutzer, sondern auch an Personen aus deren Umfeld, um die Rücklaufquote zu erhöhen. Die Stichprobe wurde mithilfe des Internets u.a. in speziellen UK-Foren und -Facebook-Gruppen akquiriert. Es beteiligten sich 115 Personen an der Onlinebefragung. Zwei Fragebögen konnten jedoch aufgrund mangelnder Angaben nicht ausgewertet werden. Vier der 113 Probanden $(3,6 \%)$ waren selbst UK-Nutzer, 75 (67,6\%) Pädagogen, 23 (20,7\%) Elternteil bzw. Angehöriger des UK-Nutzers und 9 konnten sich keiner der Gruppen zuordnen. 
Der Fragebogen war weitgehend standardisiert, d.h. er enthielt überwiegend geschlossene Fragen (z.B. Wie lange nutzt der UK-Nutzer die App MetaTalkDE?) und nur 7 offene Fragen (z. B. Welches Hilfsmittel hat der UK-Nutzer vorher verwendet?). $\mathrm{Er}$ bestand aus einer Eingangsfrage in Form einer Skalierungsfrage (Was halten Sie persönlich von dem iPad als Kommunikationshilfe? Antwort: 1-sehr sinnvoll bis 5-nicht geeignet und keine Angabe) und einem Item zur Erfassung der Probandengruppen. Im Anschluss daran wurden soziodemografische Angaben (Alter, Geschlecht, Bundesland) des UK-Nutzers erfasst. Schließlich folgten die eigentlichen Fragen zum Nutzungsverhalten in 3 Themenkomplexen:

1. Nutzung der App MetaTalkDE,

2. Nutzung des iPads allgemein und

3. Meinung zum iPad als Hilfsmittel.

Im 3. Themenkomplex wurde die Meinung der Probanden mithilfe von Skalierungsfragen erfasst (z.B. Die Multifunktionalität des iPads macht es als Kommunikationshilfe attraktiver. Antwort: 1-trifft voll und ganz zu bis 5-trifft überhaupt nicht zu und kann ich nicht einschätzen).

\section{Ergebnisse \\ $\nabla$}

Es beteiligten sich UK-Nutzer aus ganz Deutschland an der Onlinebefragung. Lediglich 52 der 113 UK-Nutzer machten vollständige soziodemografische Angaben. Demnach sind die UK-Nutzer überwiegend unter 13 Jahre alt bzw. männlich ( $\mathrm{n}=38 ; 73 \%)$, lediglich 14 (27\%) sind älter als 13 Jahre bzw. weiblich.

Alle 113 Nutzer machten Angaben zu ihrem Nutzungsverhalten. MetaTalkDE wird in vielfältiger Weise verwendet: 77 Nut- zer $(68,1 \%)$ verwenden die Rastergröße $5 \times 9, \mathrm{n}=19(16,8 \%)$ die Variante $4 \times 7$ und $17(15 \%) 3 \times 5$. Lediglich $27(28,1 \%)$ der 96 UK-Nutzer, die zusätzliche Funktionen, wie z.B. Grammatik-Popups, verwenden können, nutzen diese auch.

Das iPad wird u.a. aufgrund seiner Multifunktionalität als Kommunikationshilfe verwendet. Dies zeigte sich an den hohen Zahlen der Nutzung anderer Dinge als der App MetaTalkDE. Beispielsweise gaben 80 Nutzer (74,1\%) an, auch mit dem iPad zu spielen, und 57 (52,8\%) nutzen es auch im schulischen Kontext. Außerdem werden bestimmte Funktionen (sog. geführte Zugriffe), die das iPad bietet, von knapp der Hälfte der Nutzer (46,9\%) häufig verwendet. Die Meinung, dass das iPad die Selbstständigkeit des UK-Nutzers fördert, wird von 44 (39,3\%) der Befragten voll und ganz geteilt, 44 (39,6\%) stimmen der Aussage zu, dass es für den Nutzer die Teilhabe am Unterricht verbessert. Weiterhin geben 47 (42,3\%) der Befragten an, dass es voll und ganz zutrifft, dass die Multifunktionalität des iPads die soziale Teilhabe fördert.

\section{Fazit}

\section{$\nabla$}

Die App MetaTalkDE erfreut sich besonders großer Beliebtheit bei männlichen Nutzern unter 13 Jahre. In Verbindung mit dem iPad stellt es eine geeignete Kommunikationshilfe dar. Als Gründe für die Nutzung werden u.a. die Multifunktionalität, die technischen Möglichkeiten und die Fähigkeiten des Geräts genannt. Darüber hinaus ist aber auch relevant, dass es von vielen anderen Menschen nicht in der Funktion als Hilfsmittel verwendet wird, was einer Stigmatisierung der UK-
Nutzer entgegenwirkt und positive Auswirkungen auf die soziale Inklusion und die Lebensqualität hat. Eine Anpassung an ältere Nutzer z.B. in Form einer Wortschatzerweiterung wäre sinnvoll.

Interessenkonflikt: Die Autorin gibt an, dass kein Interessenkonflikt besteht.

\section{Literatur}

1 Braun U. Was ist Unterstützte Kommunikation? In: ISAAC, Hrsg. Handbuch der Unterstützten Kommunikation. 2. Aufl. Karlsruhe: von Loeper Literaturverlag; 2005: 01.003.001 - 01.005.001

2 Kitzinger A. MetaTalkDE Handbuch 1.6.0. 2014: Im Internet: http://www.metakidz. com/MetaTalkDE/de/MetaTalkDE.1.6.0. Handbuch.pdf (Stand: 14.09.2015)

3 Krstoski I, Reinhard S. iPad und Apps „Smarte“ Hilfsmittel für die Unterstützte Kommunikation. In: ISAAC, Hrsg. Handbuch der Unterstützten Kommunikation. Karlsruhe: von Loeper Literaturverlag; 2014: 04.063.001-04.071.001

\section{Autor}

L. Federkeil

Institut

Universität Rostock, Institut für Sonderpädagogische Entwicklungsförderung und Rehabilitation

Korrespondenzadresse

\section{Federkeil}

Budapester Straße 77

18057 Rostock

lisa.federkeil@uni-rostock.de

\section{Bibliografie}

DOI http://dx.doi.org/

10.1055/s-0041-110890

Sprache · Stimme · Gehör 2016; 40: 39-41

(c) Georg Thieme Verlag KG

Stuttgart · New York

ISSN 0342-0477 
Themenwahl Frau Lisa Federkeil hat im Rahmen ihrer empirischen Staatsexamensarbeit selbstständig und innovativ ein aktuelles Thema von hoher Relevanz für die Sonderpädagogik und insbesondere die Inklusion kommunikationsbeeinträchtigter Menschen bearbeitet. Auf der Grundlage einer stringenten und systematischen Auseinandersetzung mit dem Themenkomplex Unterstützte Kommunikation (UK) werden die Fragestellungen stringent abgeleitet.

Untersuchung Um ihre Fragestellungen zu beantworten bzw. ihre Hypothesen zur Nutzergruppe und deren Nutzungsverhalten zu überprüfen, wählte Frau Lisa Federkeil mit der Online-Benutzerbefragung eine kreative und moderne Herangehensweise, um sich die Nutzergruppe der App MetaTalkDE zu erschließen. Deutschlandweit beteiligte sich eine ungewöhnlich hohe Anzahl von insgesamt 115 Probanden, von denen allerdings die soziodemografischen Daten nur bedingt auswertbar waren. Da die Fragen zum Nutzerverhalten von der überwiegenden Mehrheit der Probanden beantwortet wurden, können die Ergebnisse als aussagekräftig bezeichnet werden. Die statistische Auswertung der Daten erfolgte mit angemessenen Methoden, war umfassend und nachvollziehbar dargestellt. Die Ergebnisse wurden hinsichtlich verschiedener Aspekte und vor dem theoretischen Hintergrund diskutiert und in den bisherigen Stand der Forschung eingeordnet.

Mehrwert Die Arbeit stellt einen wichtigen Beitrag zur Auseinandersetzung mit modernen medialen Möglichkeiten der Unterstützten Kommunikation (UK) vor allem im Hinblick auf inklusive Aspekte dar. Die Nutzung des iPads als Medium der Kommunikation führt nach den Ergebnissen der Nutzerbefragung zu einer Erhöhung der Akzeptanz und einer Verminderung von Stigmatisierungsprozessen in der sozialen Umgebung. Zudem kann die Teilhabe am Unterricht verbessert werden. Frau Federkeil leitet einige praktische Implikationen zur Verbesserung der Nutzbarkeit der App in der Altersgruppe der über 13-Jährigen und somit zu deren Weiterentwicklung ab. Das hohe Innovationspotenzial, die Selbstständigkeit bei der Durchführung der Untersuchung sowie die Auswertungen und deren sorgfältige methodenkritische Reflexion machen die Arbeit von Frau Federkeil zu einer der besten Staatsexamensarbeiten des Abschlussjahrgangs 2015 am Institut für Sonderpädagogische Entwicklungsförderung (ISER) der Universität Rostock.

Prof. Dr. Tanja Jungmann, Rostock 